Marquette University

e-Publications@Marquette

Biomedical Engineering Faculty Research and

Publications

Biomedical Engineering, Department of

2-23-2002

\title{
Estimation of Pulmonary Arterial Volume Changes in the Normal and Hypertensive Fawn-Hooded Rat from 3D Micro-CT data
}

\author{
Robert C. Molthen \\ Marquette University, robert.molthen@marquette.edu \\ Christian Wieholt \\ University of Chicago \\ Steven Thomas Haworth \\ Medical College of Wisconsin \\ Christopher A. Dawson \\ Medical College of Wisconsin
}

Follow this and additional works at: https://epublications.marquette.edu/bioengin_fac

Part of the Biomedical Engineering and Bioengineering Commons

\section{Recommended Citation}

Molthen, Robert C.; Wieholt, Christian; Haworth, Steven Thomas; and Dawson, Christopher A., "Estimation of Pulmonary Arterial Volume Changes in the Normal and Hypertensive Fawn-Hooded Rat from 3D MicroCT data" (2002). Biomedical Engineering Faculty Research and Publications. 133.

https://epublications.marquette.edu/bioengin_fac/133 


\title{
Estimation of Pulmonary Arterial Volume Changes in the Normal and Hypertensive Fawn-Hooded Rat from 3D Micro-CT Data
}

\author{
Robert Molthen ${ }^{\mathrm{a}, \mathrm{b}, \mathrm{c}, *}$, Christian Wietholt ${ }^{\mathrm{b}, * *}$, Steven Haworth ${ }^{\mathrm{a}, * * *}$, and Christopher Dawson ${ }^{\mathrm{a}, \mathrm{b}, \mathrm{c}, * * * *}$ \\ ${ }^{2}$ Department of Physiology, Medical College of Wisconsin, Milwaukee, WI, 53226, \\ ${ }^{b}$ Department of Biomedical Engineering, Marquette University, Milwaukee, WI 53203, \\ 'Zablocki VA Medical Center, Milwaukee, WI, 53295
}

\begin{abstract}
In the study of pulmonary vascular remodeling, much can be learned from observing the morphological changes undergone in the pulmonary arteries of the rat lung when exposed to chronic hypoxia or other challenges which elicit a remodeling response. Remodeling effects include thickening of vessel walls, and loss of wall compliance. Morphometric data can be used to localize the hemodynamic and functional consequences. We developed a CT imaging method for measuring the pulmonary arterial tree over a range of pressures in rat lungs. X-ray micro-focal isotropic volumetric imaging of the arterial tree in the intact rat lung provides detailed information on the size, shape and mechanical properties of the arterial network. In this study, we investigate the changes in arterial volume with step changes in pressure for both normoxic and hypoxic Fawn-Hooded (FH) rats. We show that FH rats exposed to hypoxia tend to have reduced arterial volume changes for the same preload when compared to FH controls. A secondary objective of this work is to quantify various phenotypes to better understand the genetic contribution of vascular remodeling in the lungs. This volume estimation method shows promise in high throughput phenotyping, distinguishing differences in the pulmonary hypertensive rat model.
\end{abstract}

Keywords: Pulmonary, Arterial, Volume, Remodeling, X-ray, Micro-CT, Fawn-Hooded, Rat, Hypertension

\section{INTRODUCTION}

\subsection{QuantifyingVascular Remodeling}

The rat has been used extensively for studying pulmonary vascular remodeling using histological measurements 1.23 ,

4. Hypoxia is often used as the experimental stimulus for invoking pulmonary vascular remodeling in the rat ${ }^{5,6,7,8,9,10}$. Rabinovitch et. al. histologically characterized the overall pattern of vascular remodeling in hypoxic rats as including extension of smooth muscle into small previously non-muscular arteries, medial thickening in normally muscular arteries, and a decrease in the number of arteries that filled when a barium containing contrast agent was injected at high pressure. Knowing the cellular composition of the vessel wall at discrete points along the tree provides only inferential data on how the vessel will responsed to changes in pressure or flow. While changes observed in the composition of the vessel walls obviously have important functional implications, they represent only a part of the remodeling process. It is actually the lumenal dimensions and vessel wall properties that determine the hemodynamic consequences of remodeling in the pulmonary arteries, but they are not directly available from histological measurements alone. This is emphasized by the fact that within the normal pulmonary arterial tree, the vessel wall histology varies substantially with vessel size ${ }^{11}$, but measurements of vessel distensibility, have been shown to be vessel size independent ${ }^{12,13}$. Therefore, for a more complete and useful understanding of the effects of vascular remodeling, requires additional information.

The extant lumenal morphometry of the pulmonary arterial tree comes almost exclusively from measurements on plastic corrosion casts ${ }^{14,15}$. Corrosion casts have been important in developing present concepts regarding normal pulmonary structure-function relationships ${ }^{16,17}$. However, the data collection process is very tedious, and, as a consequence, the data obtained to date are from a very small number of lungs, i.e., from only one or two ostensibly normal lungs of each studied species. This laborious method virtually precludes accumulation of data on changes in pulmonary arterial architecture resulting from vascular remodeling. As an alternative, micro $\mathrm{x}$-ray computed tomography, micro-CT, has the potential for increasing the throughput enough to make such studies practical. Key advantages include the fact that the data set, comprised of all the vessels with their correct spatial orientation and connectivity, is rapidly available in digitized form, and that the data 
can be collected on the same lung under different experimental conditions allowing for evaluation of vessel mechanics as aell as structure ${ }^{18.13}$.

\section{Pulmonary Arterial Volume}

A number of avenues can be used to estimate the pulmonary vascular volume. For example, using measurements from cast data determined by Jiang et. al. ${ }^{15}$ in which they employed a silicone elastomer (Microfil CP-101, Flow Tech, Boulder, $\mathrm{CO}$ ) and $\mathrm{Cab}-\mathrm{O}-\mathrm{Sil}$ (Eastman Kodak) to fill only the arterial system of five Sprague-Dawley rat lungs, the an estimate of arterial volume can be made by determining the average volume in each branch segment and then determining the cumulative volume by adding up all the segments in the tree. Another estimate of the arterial volume can be made using measurements of the main trunk and daughter branch diameters and lengths from CT data determined by Karau et. al..$^{22}$. In this case, using the self consistency model proposed, volume is estimated by recursively reconstructing the tree from a minimal data set then once more adding up the volume from the resulting vessel segments. In this study, we compare these methods against that of estimating the arterial volume by adding up the total number of pixels in the arterial tree within the isotropic micro-CT reconstructions and multiplying by the volume contained in each voxel. All of these estimations methods will be dependent on the accuracy of the morphometric measurements and/or the resolvability of the smaller order vessels within the tree.

\section{METHODOLOGY}

\subsection{Rat Preparations}

The rat was exposed to hypoxia using an environmental chamber. It consists of an airtight acrylic enclosure large enough to house standard rat cages. It has multiple ports through which to regulated input and output gases. A mixture of room air and nitrogen was continuously supplied to the chamber in order to maintain an oxygen concentration of (11.5\% oxygen). Total flow delivery to the chamber is 5-6 liters/minute. The chamber was opened daily for several minutes to perform cage, food, water and bedding replacement/refilling. Rats were placed in the chamber and were allowed to eat and drink ad libitum. Rats were either placed in the chamber under hypoxic conditions or in the chamber with fresh room air available under normoxic conditions ( $21.0 \%$ oxygen).

After the 21 day period, each rat was anesthetized with sodium pentobarbital $(50 \mathrm{mg} / \mathrm{kg}$ ip) and a midline sternotomy performed. The rat was heparinzed ( $200 \mathrm{IU} / \mathrm{kg}$ ) by right ventricular injection and a blood sample removed for hematocrit determination. The trachea and pulmonary artery cannulated, and the heart excised for right ventricular free wall and left ventricle plus septum weight determination. The lung was perfused with a physiological salt solution (pss) containing $5 \%$ serum albumin over a flow range of 0 to $40 \mathrm{ml} / \mathrm{min}$ to clear blood and ventilated with a $15 \% \mathrm{O}_{2}, 6 \% \mathrm{CO}_{2}$ in $\mathrm{N}_{2}$ gas mixture 3 torr end expiratory pressure and 8 torr end inspiratory pressure to eliminate any atelectasis that might have occurred during the excision. The right lobar artery was ligated and the left lung was placed in the imaging chamber.

\subsection{Lung CT Imaging and Reconstruction}

The pss was replaced by perfluorooctyl bromide (Perflubron) to obtain high intravascular $\mathrm{x}$-ray absorbance. It is important to note Perflubron does not pas into or through the capillaries, at physiologically relevant pressures, therefore only the arteries contain the contrast medium. Ventilation was then halted at a tracheal pressure of 6 torr, with the pleural pressure equal to atmospheric pressure. The arterial pressure was set at 30 torr and the lungs rotated in the $x$-ray beam at $1^{\circ}$ increments to obtain 360 planar images. The pressure was lowered successively to 21,12 , and 5 torr and then returned to 30 torr with full CT scans obtained at each pressure.

The micro-focal x-ray CT system is composed of a Fein-Focus-100.50 x-ray source (3um focal spot), a North American Imaging AI-5830-HP image intensifier coupled to a Silicon Mountain Design (SMD) CCD, and a New England Affiliated Technologies specimen micromanipulator stage all mounted on a precision rail with position information provided by Mitutoyo linear encoders. The geometry of the imaging system allows magnification of the specimen to be increased by decreasing the specimen's proximity to the $x$-ray source. Each planar projection image is a result of averaging 30 frames to minimize. The image data ( $512 \times 512$ pixels) is sent from the CCD to a frame grabber board mounted in a Dell 610 workstation running the WindowsNT operating system. Image acquisition, positional information recording and stage control are all preformed by custom written in-house window based software running on the workstation. The data is then transferred via network to a SuSE Linux 6.2 kernel 2.2.14 based Dell 410 workstation and after proper preprocessing of the 
projection images to compensate for distortions introduced by the imaging chain ${ }^{13}$, isotropic reconstructions are obtained through an implementation of the Feldkamp cone-beam algorithm ${ }^{23}$.

\subsection{Measuring Arterial Volume}

The field of view was set to maximum magnification while keeping the entire left lung in the field of view. Variability in the imaging parameters mostly reflects the differing sizes of the rat lungs imaged. In the experiments where an attempt was made to calibrate the image volume and estimate and absolute volume. The raw CT data were used after eliminating extraneous objects (the cannula or the residual right lobar artery) from the image volume. The relative change in volume over a range of pressures between the hypoxic and normoxic lungs, the values in the isotropic volumes were normalized and scaled such that each voxel represented the same physical volume. Because under these second conditions we are looking for a "change" in volume, and also have the objective of high throughput, identifying and removing unwanted object from the image volume is not required. Measurements were made in the isotropic reconstructed volume consisting of approximately $512 \times 512 \times 512$ or 134 Mega volxels.

The method used for estimating the arterial volume was based on simple grayscale thresholding techniques, and on the fact that a cannula (with a known inner diameter such that its volume can be calculated) filled with Perflubron, was included in each lung scan. The calibration procedure involved analyzing several slices through the reconstruction that includes only the cannula. The known volume in the cannula (multiple slices are used to provide larger sample) was iteratively matched (by changing the threshold value used) with that calculated by adding up the volume of the all of the voxels included over the current threshold. When the two volumes have been matched, the appropriate threshold is stored and then used on the entire reconstructed image to estimate the arterial volume.

A similar procedure was performed using a seeded region-growing algorithm ${ }^{32}$. The algorithm obtains the seed point by user interaction, where a point is specified at a location within the vessel tree. The growth of the region is controlled by specifying a grayscale window, voxel values within the window belong to the vascular tree, those outside do not. The user can also restrict the growth of the region by specifying spatial boundary values. This is to prevent "leaking" into other regions of the image volume such as the cannula or artifacts at the edges of the image volume. The seed point or voxel is entered into a list of points to be analyzed (a work list) and stored in a three dimensional binary array. The algorithm proceeds by examining the 26 neighboring voxels of the points in the work list. If a neighbor is not part of the vascular tree already, and is within the specified grayscale window it is added to the work list, and marked as part of the tree in the binary array. The algorithms terminates when all entries in the work list have been examined. Therefore the algorithm grows the tree in all directions stopping when the voxel values are not within the windowed limits or the set boundaries have been approached. To obtain a volume estimate the marked voxels in the binary array are counted. The region growing algorithm was implemented in this study as a way to verify that results of the simple thresholding method were comparable such that disconnected regions of the image were not being included as part of the arterial tree erroneously.

In calculating arterial volume from the principal pathway data lumenal measurements were made in arteries ranging in size from approximately $80-1700 \mathrm{um}$, see figure 1 . Measurement of only the main trunk or longest principal pathway is justified provided that the assumption based on the "self-consistency" property holds true for the arterial branching structure $^{13,22}$. The notion of a self-consistent tree holds important implications about how much of the vascular tree must be measured in order to completely characterize the structure and function of the vascular network. A tree that satisfies the conditions of self-consistency will display the property that all branches of the vascular tree are statistically indistinguishable from their respective sub-regions of the main trunk, where their respective sub-regions are defined by a point at which the main trunk's diameter equals the inlet diameter of the branch vessel and includes the rest of the main trunk distal to this point. . For example, in figure 1 , this can be thought of as sliding a marker representing a daughter branch (circles) horizontally to the right until it intersects the curve representing the main trunk (squares) and then replacing the daughter with a copy of the main trunk segment (with the closest diameter) and all its distal branches. In a tree where the selfconsistency condition is met, the monumental feat of measuring all the vessels in the pulmonary tree is reduced to measuring only the main pulmonary trunk and the inlet diameter of the daughter branches along the main trunk. Self-consistency provides a means for summarizing the global morphometric and mechanical properties of the vascular tree from a reduced set of measurements. Additional measurements of the same lung at several different intravascular pressures were made at identical locations in the trunk. A computer algorithm was written in Matlab version 6, which reconstructs an entire pulmonary tree from the principal pathway data building from the distal vessels to the lobar artery. The algorithm involves recursively replacing each missing daughter branch with all portions of the main trunk distal to a region having the closest diameter and adding up the segment volumes in the process. 


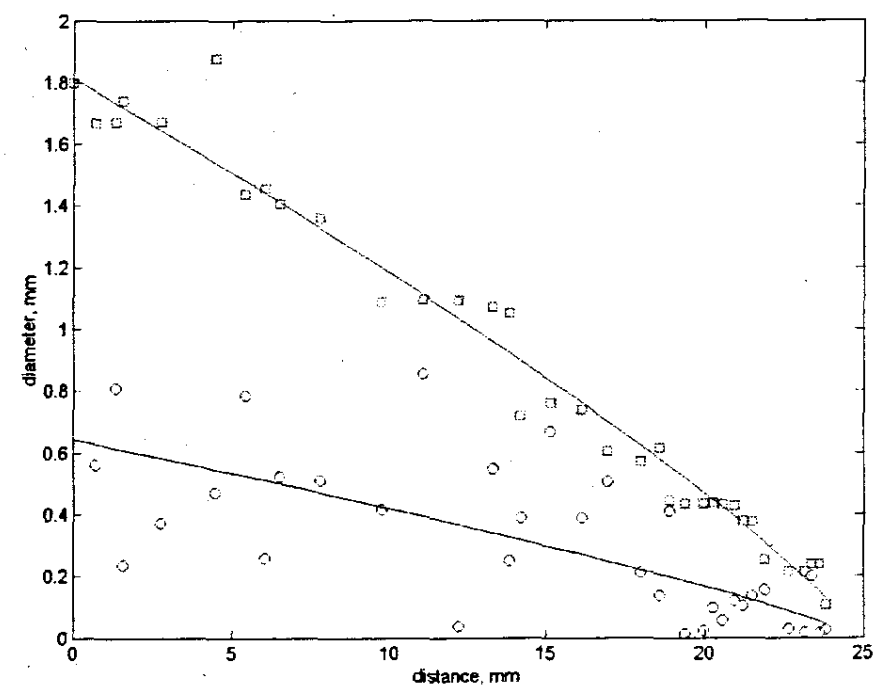

Fig. 1: Principle pathway data for a rat left lung. Data points represent diameters vs. distance from inlet for principal path segments. Squares, main arterial trunk. Circles, daughter branches.

\section{RESULTS}

\subsection{Visualizing the Effects of Hypoxia Induced Remodeling}

Surface shaded renderings for left lung arterial trees from hypoxic and normoxic rat are shown in figures 2 and 3. Since the rendering method involves thresholding, small vessels seen at high pressure are not included in the lower pressure renderings as their grayscale values fall below the threshold set. The rendering threshold was chosen to reveal the underlying tree structure. The lower compliance of the hypoxic pulmonary arteries is seen as the relative reduced distention.

Calibrated measurement of the vascular volume as describe in the methodology section was done on a normoxic full lung and a hypoxic and normoxic left lung lobes. Calibration to the cannula volume requires scanning the grayscale threshold value while comparing the known cannula volume to that calculated from the CT volume. Figure $4 \mathrm{a}$ is a graph of the apparent cannula volume (one slice) vs. the grayscale threshold value. Figure $4 \mathrm{~b}$ relates how a similar threshold scan would effect the calculated volume of the entire arterial tree. Figure 5 shows the binary image of the $225^{\text {th }}$ transaxial slice through an intact full rat lung created using grayscale thresholds of $0.5,1.1,1.8, a-c$, respectively, (intravascular pressure approximately 11.6 torr). Figure $5 \mathrm{~d}$ shows the same slice from a volume created using the seeded region growing technique. The difference between the images in $5 \mathrm{~b}$ and $5 \mathrm{~d}$ are imperceptible. In the full rat lung, the cannula volume matched the volume calculated through thresholding at a grayscale of 1.1 .

\subsection{Volume Measurements}

The estimates of arterial volume vs. intravascular pressure using the cannula calibration scheme are shown for a normoxic full rat lung in table 1 and for both normoxic and hypoxic left rat lungs in figure 6. Table 1 also gives the volume calculated through implementation of the region growing algorithm. The number from both methods are close suggesting that very few pixels within the image volume and above the set threshold are isolated from the arterial tree. This result confirms that the thresholding method is reasonable for selectively segmenting out the arterial tree while not including extraneous pixels. Figure 6 highlights the differences between the two experimental conditions. The normoxic lobe experiences a larger volume change than the hypoxic lung lobe, and has a smaller volume at 5.4 torr and a larger volume at 30 torr.

A typical physiological pressure in the pulmonary artery of a rat is around 13 torr. One of the four pressures that was used in our imaging protocol was in this range (12 torr) and the segment data presented by Jiang was also in this range (14.3 torr). In this pressure range, the estimated values of left lung pulmonary arterial volume arrived at through several different methods of calculation, are shown in Table 2. The first method is the above cannula calibration and thresholding. 

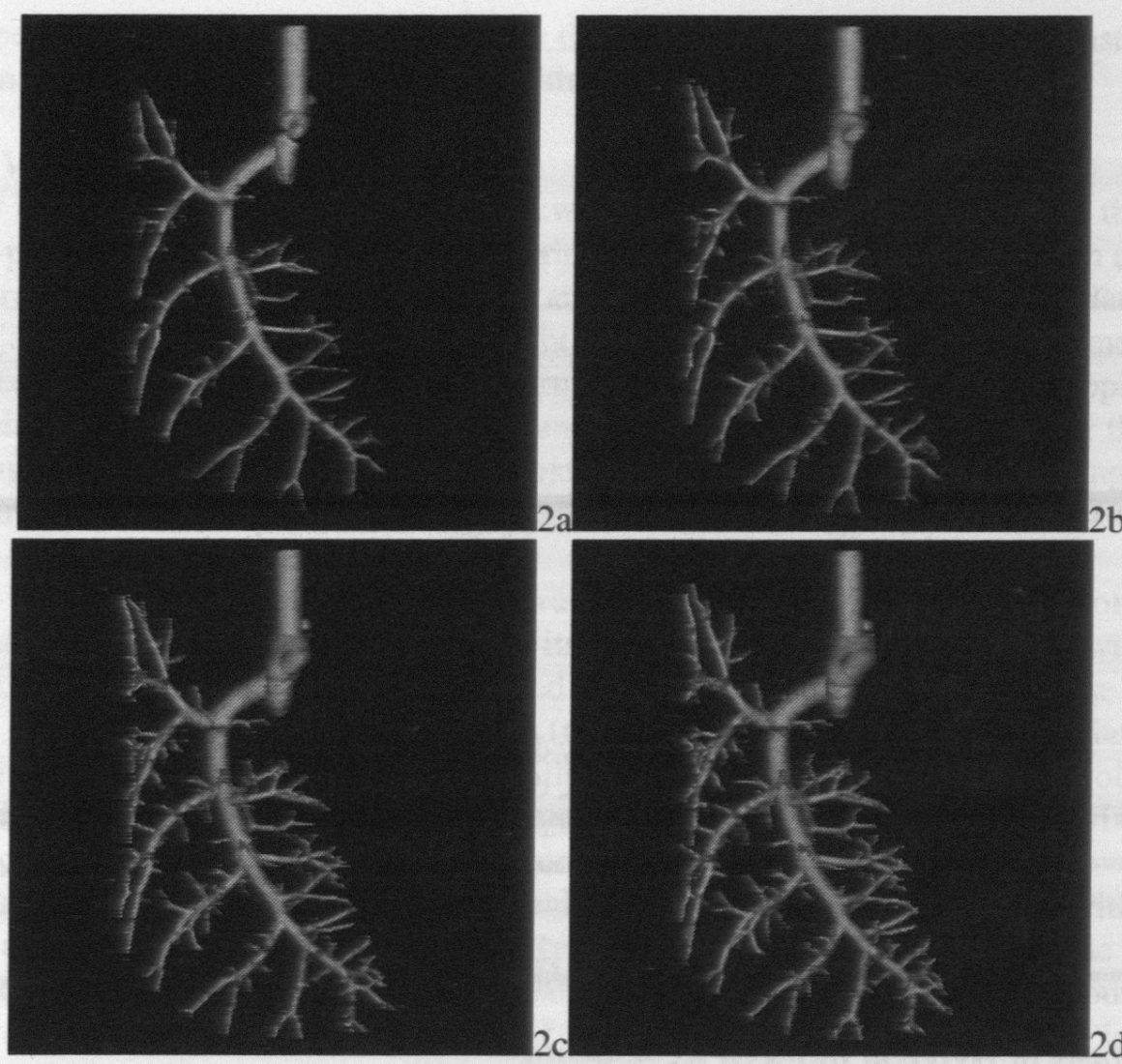

Fig. 2: Surface shaded renderings of arteries in a hypoxic left lung imaged at 5.4, $12,21,30$ torr, a-d respectively (field of view $3.47 \mathrm{~cm}$ ).
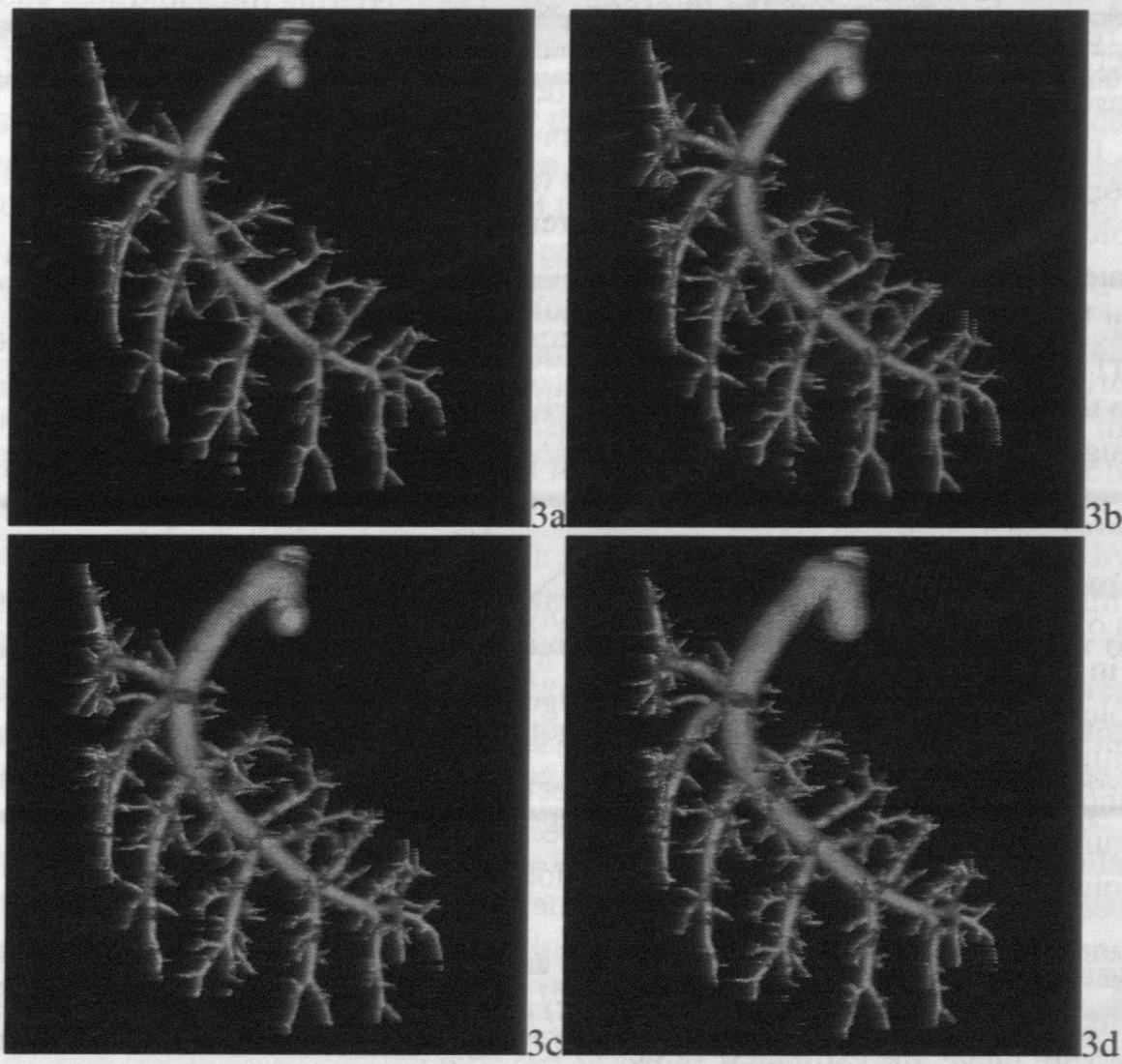

Fig. 3: Surface shaded renderings of arteries in a normoxic left lung imaged at $5.4,12,21,30$ torr, a-d respectively (field of view $2.83 \mathrm{~cm}$ ). 

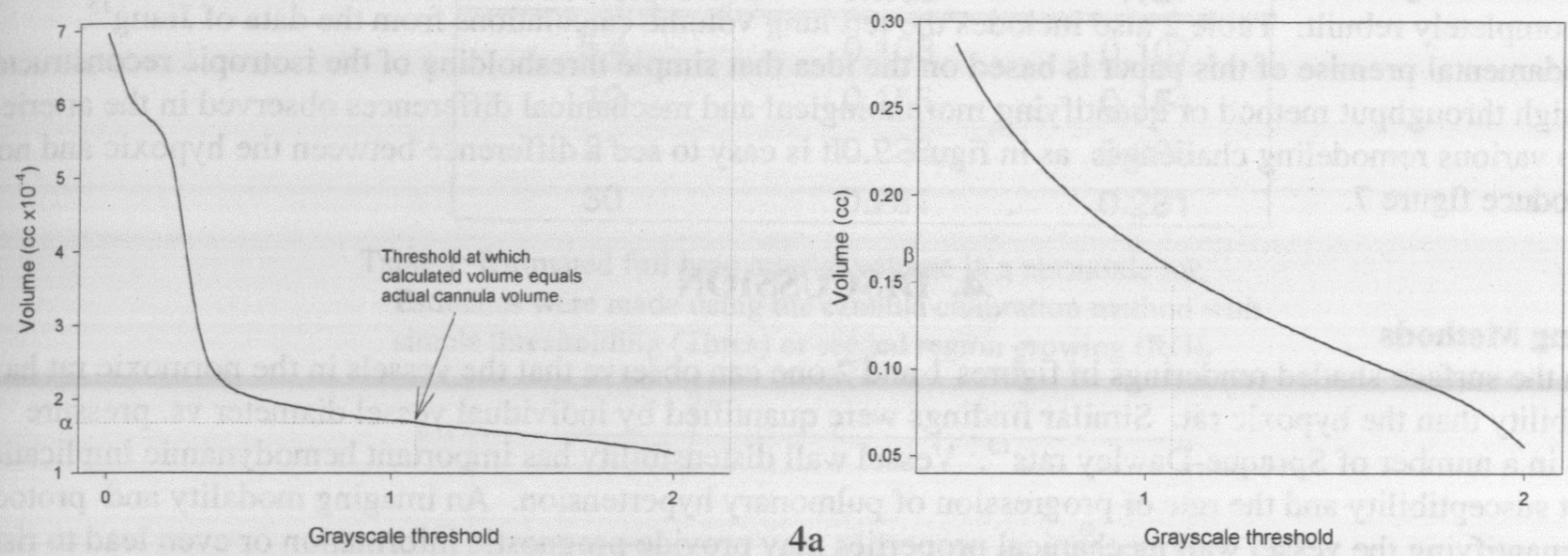

Fig. 4: a) Grayscale threshold vs. calculated cannula volume. $\alpha=$ cannula volume. b) Grayscale threshold vs. pulmonary arterial volume in a rat (full) lung. $\beta=$ arterial volume.

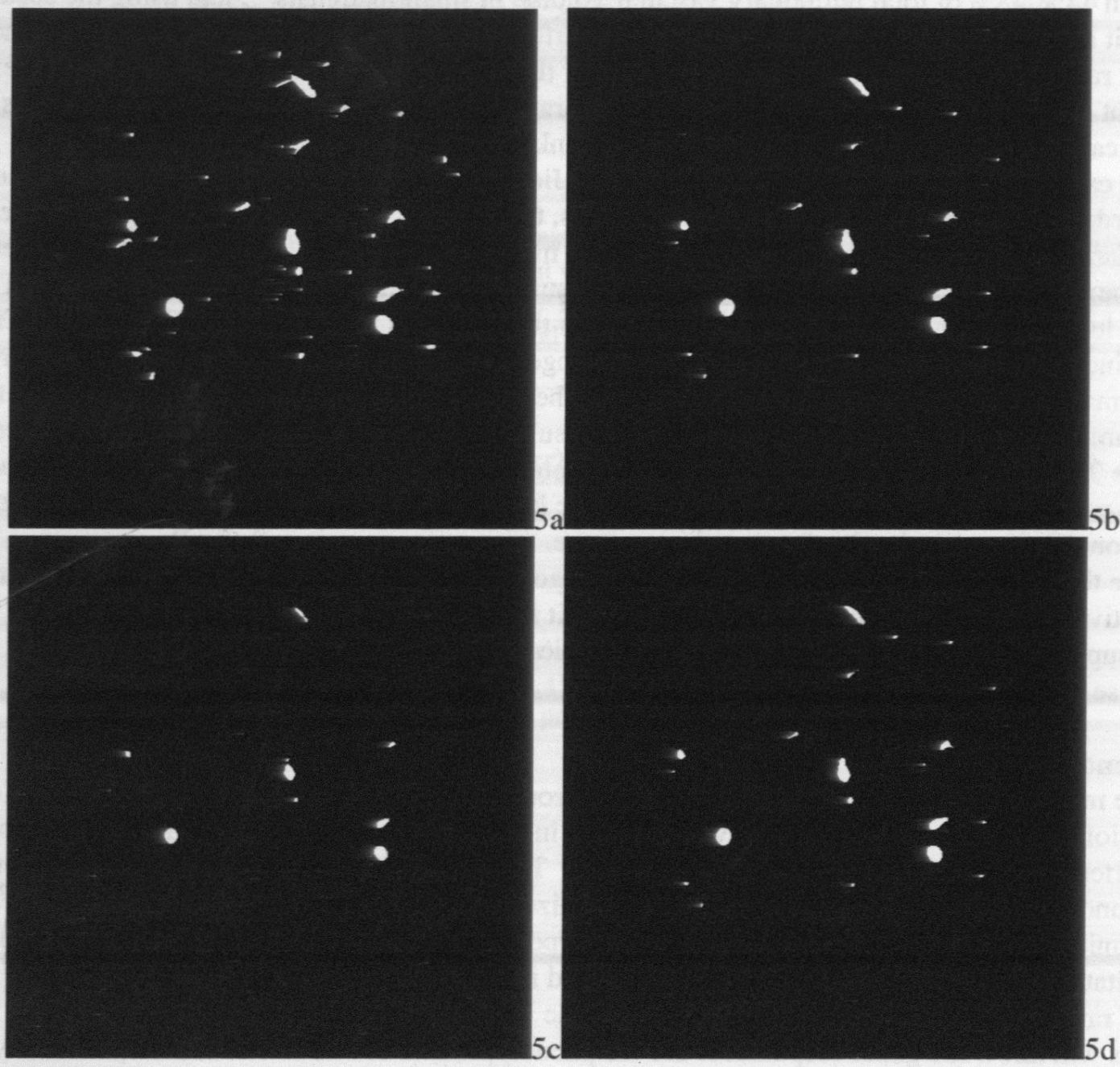

Fig. 5: Binary image of the 225th transaxial slice through an intact rat (full) lung with intravascular pressure at approximately r1.6 torr. The binary images are created by thresholding the reconstructed volume at grayscale values of $0.5,1.1,1.8, a-c$ respectively. Fig. $d$ was created by performing seeded region growing with the lower grayscale threshold of 1.1.

Field of view $=3.8 \mathrm{~cm}$. 
The second method, discussed in section 2.3 above, involves reconstructing the arterial tree from the principal pathway data, calculating the total arterial volume by recursively reattaching and summing up distal branch volumes until the tree has been completely rebuilt. Table 2 also includes the left lung volume calculations from the data of Jiang ${ }^{15}$.

A fundamental premise of this paper is based on the idea that simple thresholding of the isotropic reconstructed data will lead to a high throughput method of quantifying morphological and mechanical differences observed in the arteries of rats exposed to various remodeling challenges, as in figure 7 . It is easy to see a difference between the hypoxic and normoxic rats used to produce figure 7.

\subsection{Comparing Methods}

\section{DISCUSSION}

From the surface shaded renderings in figures 1 and 2 one can observe that the vessels in the normoxic rat have a larger distensibility than the hypoxic rat. Similar findings were quantified by individual vessel diameter vs. pressure measurements in a number of Spraque-Dawley rats ${ }^{13}$. Vessel wall distensibility has important hemodynamic implications and may affect susceptibility and the rate of progression of pulmonary hypertension. An imaging modality and protocol that is capable of quantifying the vessel wall mechanical properties may provide prognostic information or even lead to risk assessment and early detection.

Previous estimates of the total capillary blood volume in the pulmonary circuit of the rat are approximately $.66 \mathrm{~mL}$ reported by Crapo and $.48 \mathrm{~mL}$ by Weibel using the morphometric techniques of Weibel ${ }^{19,} 20$. With estimates of capillary volume ranging from $44 \%-48 \%$ of total pulmonary vascular volume in small mammals ${ }^{21}$, and using the assumption that the blood volume is split approximately in half between veins and arteries of the lung, we can estimate the pulmonary arterial volume in the intact rat lung to be between .31 and $.44 \mathrm{~mL}$. For the full rat lung our results, see Table 1 , seem to fall close to these values. In light of the discussion of how the cannula calibration method tends to exclude small vessels, we would expect the volumes calculated to be the lower bounds on the trunk data.

In order to calculate the left lung arterial volume from Jiang's data obtained from plastic casts ${ }^{15}$, the tree has been broken down into orders where the average number of segments, the average length and the average diameter are given for every order. Calculating volume for the model lung is simply a matter of finding the volume for each diameter/length pair, multiplying by the number of branches for each order and then summing up the volume across the orders. The value calculated for a left lung lobe, see Table 2, was well in line with the estimates made through CT imaging. The calculations of volume from the principal pathway data are also in the same range. Given the tree rebuilding process required for this method and the estimation of a daughter branch replacement, whether we use a portion of the main trunk with slightly larger or slightly small diameter, can have significant effects on the resulting down stream volume. It is also interesting to note that in figure 6 and table 2, near the typical physiological range for pulmonary arterial pressure, about 13 torr, the volume measurements are very close for both the normoxic and hypoxic lung. This may be a hint of an underlying mechanism which acts to maintain a constant arterial blood volume at this pressure.

The simple thresholding and seeded region growing algorithm, show nearly identical results. The region growing technique conservatively includes less than $1 \%$ fewer pixels as it insures direct connectivity with the rest of the pixels in the arterial tree. This supports the use of the faster method of simple thresholding, at least in detecting the balk structural changes seen in these hypoxia studies.

\subsection{Functional Genomics}

One of the motivations for the use of CT is for high throughput phenotyping for functional genomics and its subdiscipline, physiological genomics ${ }^{24,25.31}$ as new paradigms in biological research. The FH rat has a genetic susceptibility to pulmonary hypertension in response to chronic hypoxia ${ }^{26,27}$. The utility of studying rats with specific genetic backgrounds for physiological/functional genomics has recently been recognized and is a fast growing field of research ${ }^{28,25}$. One tool of physiological genomics is the utilization of quantiative phenotypes and high resolution genetic mapping to identify regions of the genome (quantitative trait loci (QTL)) where genes involved in phenotypes are located ${ }^{29,30}$. The use of consomic and congenic strains of rats in studies such as this one, will allow the identification of phenotypical traits. QTL and other methods of analysis will allow the genes responsible for the phenotypic trait to be localized, with previously unknown functions being identified by using the ever expanding cDNA library and ultimately the entire genetic sequence.

Thus, the proposed method of arterial volume estimation, is also proposed as a relatively high throughput method for identifying a quantitative phenotypic trait and to some degree should reflect global changes in pulmonary vessel elasticity. 


\begin{tabular}{|c|cc|}
\hline & \multicolumn{2}{|c|}{ Volume (cc) } \\
Pressure (torr) & Thres & RG \\
\hline 5.4 & 0.108 & 0.107 \\
12 & 0.155 & 0.154 \\
21 & 0.229 & 0.229 \\
30 & 0.281 & 0.281 \\
\hline
\end{tabular}

Table 1: Estimated full lung arterial volume in a normoxic rat.

Estimates were made using the cannula calibration method with simple thresholding (Thres) or seeded region growing (RG).

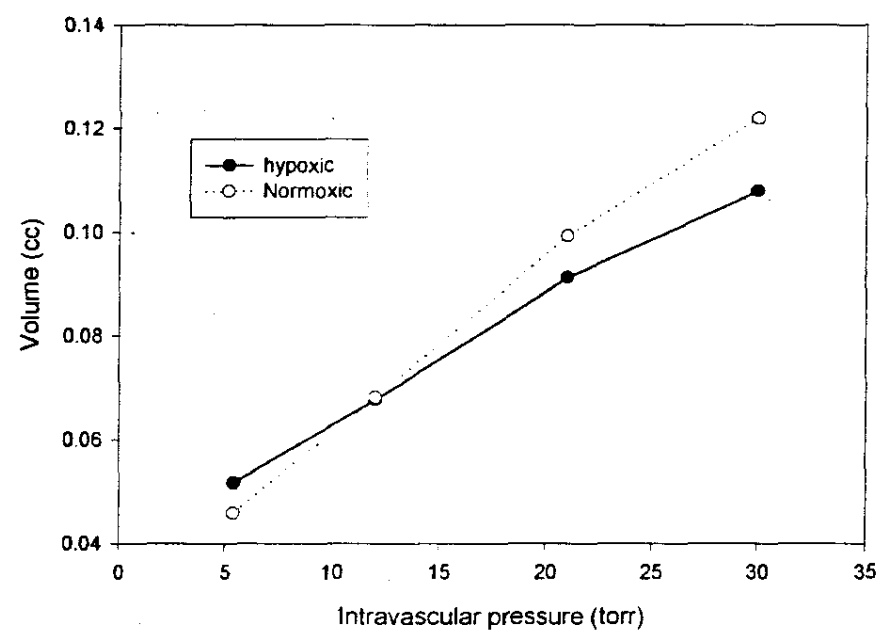

Fig 6: Estimated left lung arterial volume in a chronically hypoxic conditioned and normoxic rat. Estimates were made using the cannula calibration method and normalized for body weight.

\begin{tabular}{|l|c|cc|}
\hline & & \multicolumn{2}{|c|}{ Volume(cc) } \\
\cline { 3 - 4 } i Method & Pa (torr) & Hypoxic & Normoxic \\
\hline \hline calibrated thres & 12 & 0.0678 & 0.0682 \\
Jiang et al [15] & 14.3 & N/A $^{*}$ & $0.069^{*}$ \\
pathway data & 12 & 0.0529 & 0.0851 \\
\hline
\end{tabular}

Table 2: Estimated left lung arterial volume in a chronically hypoxic conditioned and normoxic rat. (*data only available for control). Pathway data calculated from principal pathway data. 


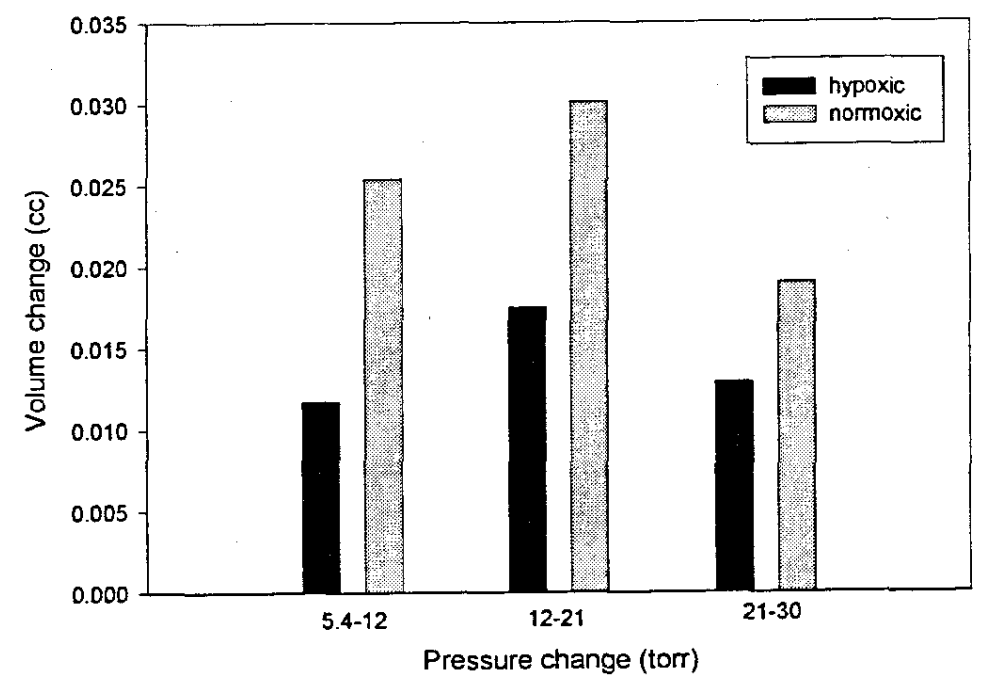

Fig 7: Estimated left lung arterial volume change in a chronically hypoxic conditioned and normoxic rat.

\section{ACKNOWLEDGMENTS}

This work supported by HL 19298, the Whitaker Foundation, the W.M. Keck Foundation and the Department of Veterans Affairs. I would also like to thank Gary Krenz, Christopher Hangar, Anita Dhyani, Kelly Karau, and David Roerig for their contributions to this study.

\section{REFERENCES}

1. DAVIES, P, F MADDALO, AND L REID. Effects of chronic hypoxia on structure and reactivity of rat lung microvessels. J. Appl. Physiol. 58: 795-801,1985.

2. MEYRICK, B, AND L REID. Hypoxia and incorporation of 3H-Thymadine by cells of the rat pulmonary arteries and alveolar wall. Am. J. Path. 96: 51-70,1979.

3. MEYRICK, B, W GAMBLE, AND L REID. Development of crotalaria pulmonary hypertension: Hemodynamic and structural study. Am. J. Physiol. 239: H692-H702,1980.

4. Rabinovitch, M, MA KonstaM, WJ Gamble, N PAPANicolaou, MJ ARonovitz, S Treves, AND L ReID. Changes in pulmonary blood flow affect vascular response to chronic hypoxia in rats. Circ. Res. 52: 432-441,1983.

5. HEROLD, CJ, RC WETZEL, JL ROBOTHAM, SM HEROLD, AND EA ZERHOUNI. Acute effects of increased intravascular volume and hypoxia on the pulmonary circulation: Assessment with high-resolution CT1. Radiology 183: 655-662,1992.

6. EDDAHIBI, S, B RAFFESTIN, M CLOZEL, M LEVAME, AND S ADNOT. Protection from pulmonary hypertension with an orally active endothelin receptor antagonist in hypoxic rats. Am. J. Physiol. 268 (Heart Cir. Physiol. 37):: H828-H835,1995.

7. ONO, S, JY WESTCOTT, AND NF VOELKEL. PAF antagonists inhibit pulmonary vascular remodeling induced by hypobaric hypoxia in rats. J. Appl. Physiol. 73: 1084-1092,1992.

8. STANBROOK, HS, KG MORRIS, AND IF MCNURTRY. Prevention and reversal of hypoxic pulmonary hypertension by calcium antagonists. Am. Rev. Respir.Dis 130: 81-85,1984.

9. PARTovian, C, S AdNot, S EdDahibi, E Teiger, M Levame, P Dreyfus, B RAFFestin, AND C Frelin. Heart and lung VEGF mRNA expression in rats with monocrotaline- or hypoxia-induced pulmonary hypertension. Am. J. Physiol. 275 (Heart Circ. Physiol. 44): H1948-H1956,1998.

10. VOELKEL, NF, AND RM TUDER. Hypoxia-induced pulmonary vascular remodeling: a model for what human disease? $J$. Clin. Invest. 106: 733-738,2000.

11. SASAKI, SI, N KOBAYASHI, T DAMBARA, S KIRA, T Sakai, Structural organization of pulmonary arteries in the rat lung, Anat Embryol, 191:477-489, 1995.

12. AL-TINAWI, A, JA MADDEN, CA DAWSON, JH LINEHAN, DR HARDER, AND DA RICKABY. Distensibility of small arteries of the dog lung. J. Appl. Physiol. 71: 1714-1722,1991. 
13. KARAU, KL, RH JOHNSON, RC MOLTHEN, AH DHYANI, ST HAWORTH, CC HANGER, DL ROERIG, AND CA DAWSON. Microfocal X-Ray CT imaging and pulmonary arterial distensibility in excised rat lungs. Am. J. Physiol. Heart Circ. Physiol.: 281: H1447-H1457,2001.

14. HORSFIEID, K. Morphometry of small pulmonary arteries in man. Circ. Res. 42: 593-597,1978.

15. JIANG, Z, GS KASSAB, AND YC FUNG. Diameter-defined Strahler system and connectivity matrix of the pulmonary arterial tree. J. Appl. Physiol. 76: 882-892,1994.

16. BENNETT, SH, BW GOETZMAN, JM MILSTEIN, AND JS PANNU. Role of arterial design on pulse wave reflection in a fractal pulmonary network. J. Appl. Physiol. 80: 1033-1056,1996.

17. DAWSON, CD, GS KRENZ, AND JH LINEHAN. Complexity and Structure-Function relationships in the pulmonary arterial tree, Chapter 13. In: Lung Biology in Health and Disease, Complexity in Structure and Function of the Lung ed., edited by MP Hlastala, and Robertson, HT. New York: Marcel Dekker, inc., 1998, p. 401-427.

18. LU, YH, EA HOFFMAN, AND EL RITMAN. Measurment of three-dimensional anatomy and function of pulmonary arteries with high-speed X-ray computed tomography. Invest. Radiol. 22: 28-36,1987.

19.CRAPO, J., BARRY, B., FOSCUE, H., SHELBURNE, J., Structural and Biochemical Changes in Rat Lungs Occurring During Exposure to Lethal and Adaptive Doses of Oxygen, American Review of Repiratory Disease, 122: 123-143, 1980. 20. WEIBEL, ER, Morphometric Estimation of Pulmonary Diffusion Capacity, I. Model and Method, Respir Physiol, 11 : $54-$ $75,1970$.

21. AUDI, S, JH LINEHAND, GS KRENZ, CA DAWSON, SB AHLF AND DL ROERIG, Estimation of the capillary transport function in isolated rabbit lungs, J.Appl. Physiol, 78(3): 1004-1014, 1995.

22. KARAU, KL, RC MOLTHEN, AH DHYAN, ST HAWORTH, CC HANGER, DL ROERIG, RH JOHNSON AND CA DAWSON. Pulmonary arterial morphometry from microfocal X-ray computed tomography. Am. J. Physiol. Heart Circ. Physiol.: 281: H2747-H2756,2001.

23. FEL DKAMP, LA, LC DAVIS, AND JW KRESS. Practical cone-beam algorithm. J. Opt. Soc. Am. A 1: 612-619,1984.

24. COWLEY, AW The emergence of physiological genomics. J. Vasc. Res. 36: 83-90,1999.

25. COWLEy, AW, M STOLL, AS GREENE, ML KALDUNSKI, RJ ROMAN, PF TONELlATO, NJ SCHORK, P DUMAS, AND HJ

JACOB. Genetically defined risk of salt sensitivity in an intercross of Brown Norway and Dahl S rats. Physiol. Genomics 2: 107115,2000 .

26. SATO, K, S WEBB, A TUCKER, M RABINOVITCH, RF O'BRIEN, IF MCMURTRY, AND TJ STEI ZNER. Factors influencing the idiopatic development of pulmonary hypertension in the fawn hooded rat. Am. Rev. Respir.Dis 145: 793-797,1992.

27. LECRAS, TD, DH KIM, S GEBB, NE MARKHAM, JM SHANNON, RM TUDER, AND SH ABMAN. Abnormal lung growth and the development of pulmonary hypertension in the Fawn-Hooded rat. Am. J. Physiol. 277 (Lung Cell. Mol. Physiol. 21): L709L718,1999.

28. BRown, DM, TC MATISE, G. KoIKE, S ZANGEN, JS SIMON, MG MCLAUGHLIN, ES WINER, A CHAKRAVARTI, ES LANDER, HJ JACOB. An integrated genetic linkage map of the laboratory rat. Mammalian Genome. 9: 521-530, 1998.

29. NADEAU, JM, JB SINGER, A MATIN, AND ES LANDER. Analysing complex genetic traits with chromosome substitution strain. Nature Genetics 24: 221-225, 2000.

30. EWART, SL, D KuPERMAN, E SCHADT, C TANKERSLEY, A GRUPE, D SHUBITOWSKI, G PELTZ, AND M WILLS-KARP. Quantitative trait loci controlling allergen-induced airway hyperresponsiveness in inbred mice. Am. J. Respir. Cell Mol. Biol. 23: 537-545,2000.

31. DZAU, VJ, MJF AUSTIN, P BRown, A COWLEy, D Housman, R MULUIGAN, AND R ROSENBERG. Revolution and renaissance (editorial). Physiol. Genomics 1: 1-2,1999.

32. FIEBICH M, C WIETHOLT, BC RENGER, SG ARMATO, KR HOFFMANN, D WORMANNS, S DIEDERICH Automatic detection of pulmonary nodules in low-dose, screening thoracic CT examinations. In: Hanson KM (Hrsg) Image Processing, Proc SPIE, S. 1434-1439, 1999.

* Robert.molthen@marquette.edu; phone (414) 384-2000 x41443; fax (414) 384-0115; http//semblance.bien.mu.edu/ molthen, Zablocki VA Medical Center, Milwaukee, WI, 53295,

** Christian.wietholt@marquette.edu

***Shaworth@mcw.edu,

**** cdawson@mcw.edu. 